\title{
Modelling, Simulation and Control of 5 Axis Industrial Robot using MATLAB
}

\author{
P. Vivekanandan*, A. Vishnu, Sathees Kumar Narayanasamy and R. Yasodharan \\ Department of Mechatronics Engineering, SNS College of Technology, Coimbatore - \\ 641035,Tamil Nadu, India; hodmct@snsct.org, vishnu181a@gmail.com, \\ satheeeskumaran@rediffmail.com, yaso.snstech@gmail.com
}

\begin{abstract}
Objectives: This paper introduces a model of five pivot robot arm and its re-enacted trajectory. This sort of robot is quick, precise, solid and easy to program. Robot arm has sensors and is intended for taking care of articles without human intercession. Methods/Statistical Analysis: Exhibited robot has five degrees of opportunity, every one of his developments are turning. For modelling the robot arm we utilized SolidWorks programming and for direction re-enactment, we utilized Matlab and Simulink software. Findings: A common problem faced by educational institutions concerns the limited availability of expensive robotics equipment, with which students in the improving program can work, in order to acquire valuable "hands-on" experience. Therefore, the Robot along with suitable Simulation Software is very important paramount. This paper presents the development of a visual software package where VI5SNS robot has been taken as a case study. It utilizes Mat lab/Simulink as the tools for testing motional characteristics of the VI5SNS robot. A robot model will be developed; the Forward, Inverse Kinematics, Velocity Kinematics "Jacobian" and Path Planning problems will be implemented and tested. The developed package will be used as an educational tool. Application/Improvements: This work will increase the education, training, research and development possibilities for robotics classes in graduate and under graduate studies.
\end{abstract}

Keywords: 5-Axis, Educational robot, End Effector, Kinematics, Robot, Mat Lab, Path Planning

\section{Introduction}

Robotics is an emerging technology that bypasses the old-fashioned engineering methods. In order to know the complexity function of robots and their usage involves knowledge of mechatronics system which involves mathematics, mechanical engineering, electrical and electronics engineering systems, industrial engineering, computer science and economics. Modern engineering disciplines, such as mechatronics engineering, automation engineering, applications engineering, and knowledge engineering have emerged to deal with the complexity of the field of robotics and industrial automation. This work is concerned with the basics of robotics, including anatomy, kinematics, dynamics, Jacobian, path planning, interface and control. Our aim is to include the concepts in these subjects as applied to robot manipulators, end effectors, mobile robots, and other such mechatronics systems. The majority of robot applications deal with end effector of the robot operating in well framed industrial environment. There is a large amount of literature which discuss the robot molding and analysis of industrial robots. The majority of their importance comes from discussing the low-cost educational robot arms. A visual software program will be also developed to show the robot arm motion with respect to its mathematical analysis.

\subsection{Robotic Arm}

A robot arm is a robot manipulator, usually programmable, with similar features to a man or woman arm. The hyperlinks of such a manipulator are related by way of joints allowing both rotational movement (including in a

*Author for correspondence 
articulated robotic) or translational (linear) displacement. The hyperlinks of the manipulator may be considered to form a kinematic chain. The business company end of the kinematic chain of the manipulator is referred to as the conclusion effector and it is analogous to the human hand ${ }^{1}$. The stop effector may be designed to execute any desired project such as for instance welding, gripping, spinning and so on, with respect to the utility. The robot fingers may be self-sustaining or controlled manually and can be used to execute many distinctive obligations with notable accuracy. The robot arm can be fixed or mobile (i.e., Wheeled) and can be designed for industrial or domestic programs. It is believed that maximum joints rotate no greater than a hundred and eighty tiers, due to the fact most servo automobiles cannot exceed that amount ${ }^{2}$.

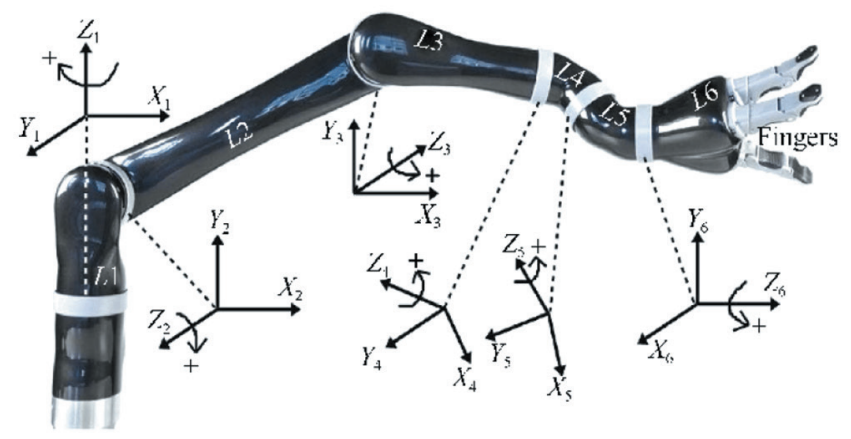

\section{Literature Review}

De Xu et al., proposed an analytical solution for a 5-DOF manipulator to check out sure trajectory while preserving the orientation of simply one axis in the end-effector body. The forward kinematics and inverse kinematics for a 5-DOF manipulator are analysed systemically. The singular hassle is mentioned after the ahead kinematics is supplied. For any given accessible position and orientation of the stop-effector, the derived inverse kinematics will provide an accurate answer. Put surely, there exists no singular trouble for the 5-DOF manipulator, which has extensive software regions consisting of as an instance welding, spraying, and Painting. BakiKoyuncu et al., presented the theoretical heritage for the analysis of the five DOf Lynx-6 educational Robot Arm kinematics. The kinematics problem is described while the transformation from the Cartesian area to the joint area and vice versa.

The Denavit-Harbenterg (D-H) sort of illustration is hired to version robot links and joints in this have a look at. Both ahead and inverse kinematics solutions for this educational manipulator are supplied, an powerful technique is suggested to lower more than one answers in inverse kinematics. An aesthetic software package, named MSG, may be advanced for checking out Motional Characteristics of the Lynx-6 Robot arm. The kinematics answers of this system package deal were determined to be same with the robot arm's physical motional behaviors ${ }^{3}$.

Mohammad Amin Rashidifar et al., proposed the Modelling and manipulate of five Degree of Freedom (DOF) robot arm is the subject of this text. The modelling hassle is important before applying manipulate strategies to guarantee the execution of any task consistent with an excellent input with minimal errors.

Deriving each ahead and inverse kinematics is an vital step in robotic modelling based on the Denavit Hartenberg $(\mathrm{DH})$ representation. Proportional Imperative spinoff (PID) controller is hired as a guide benchmark to evaluate its outcomes with fuzzy common sense controller (FLC) and Fuzzy Supervisory Controller (FSC) results. FLC is carried out as any other controller due to the nonlinearity inside the robotic manipulators. We compare the effect of the PID controller and FLC effects almost about time reaction specs. FSC is absolutely a hybrid among the previous controllers. The FSC is beneficial for tuning PID gains for the reason that PID alone performs no longer first-class in nonlinear structures. Hence, evaluation of tuning of PID parameters is applied using classical technique and FSC approach. Based on simulation effects, FLC gives higher results than classical PID controller with regards to time response and FSC is higher than classical strategies which include for example Ziegler-Nichols (ZN) in tuning PID parameters almost about time response $e^{4}$.

Vivek Deshpande et al., proposed that the manipulate of an automated arm is a huge project on the grounds that earlier days of robots. The kinematics problem is defined while the transformation from the Cartesian area to the joint space and vice versa. This paper pursuits to version the forward and inverse kinematics of a five DOF Robotic Arm for brief choose and region software. A general D-H representation of ahead and inverse matrix is received. An analytical solution for the forward and inverse kinematics of $5 \mathrm{DOF}$ robotic arm provided, to research the movement of arm from one point in area to a distinctive factor. The 5 DOF robotic arm is a vertical articulated robot, with five revolute joints. It's a straightforward and safe robot machine created for instructional motive $e^{5}$. This versatile system allows college students to attain theoretical and sensible experience in robotics, automation and 
manipulate systems. C.A. Acosta Calderon et al., proposed that the principle hassle in trajectory generation and monitoring of robot manipulators is to plan the trajectory and compute the required joint angles. Inverse kinematics modelling is generally adopted, though sometimes other approaches are needed because of the shortage of reliability and accuracy of analytical methods.

This paper offers a comparison among an analytical inverse kinematics based hybrid approach and a Resolve Motion Rate Control approach for controlling the Pioneer Arm installed at the Pioneer cell robotic. Himanshu Chaudhary et al., offered two processes to generating such trajectories: instantly traces in joint area and immediately lines in Cartesian space had been mentioned. This is one of the most common necessities in robotics for transferring the stop-effector easily from initial Region to reason area ${ }^{6}$. These are known respectively as joint space and Cartesian area tracking. Two client described algorithms are developed for Joint space in addition to Cartesian space trajectory tracking. The set of rules has been tested in simulation yielding truthful consequences, that have also been in comparison with the ones provided via another essential trajectory making plans approach strategies ${ }^{?}$.

\section{Gadget Description}

Our VI5SNS robotic has 5 instructions of movement (DOF) plus a grip movement (five +1 ) as shown in Discern 1. It is likewise similar to human arm from the number of joints factor of view. These joints offer shoulder rotation, shoulder to and fro motion, elbow movement, wrist up and down motion, wrist rotation and gripper movement ${ }^{8}$.

VI5SNS has five rotational joints and a moving grip (quit effector). Joint one represents the shoulder and its axis of movement is $\mathrm{z} 1$. This joint gives a rotational $\Theta 1$ angular motion around $\mathrm{z} 1$ axis in xly1 aircraft. Joint is diagnosed as the upper arm and its axis is perpendicular to Joint one axis.

It offers a rotational $\Theta 2$ angular movement around z2 axis in $\mathrm{x} 2 \mathrm{y} 2$ aircraft and $\mathrm{z} 3$ axes of Joint three (Forearm) and Joint 4 (Wrist) are parallel to Joint $\mathrm{z}$-axis; they provide $\Theta 3$ and $\theta 4$ angular motions in $x 3 y 3$ and $x 4 y 4$ planes respectively. Joint 5 is identified as the grip rotation. Its $z 5$ axis is vertical to $\mathrm{z} 4$ axis and it offers 95 angular motions in $\mathrm{x} 5 \mathrm{y} 5$ aircraft. The end effector is a replaceable one, in which it could be modified consistent with the packages?

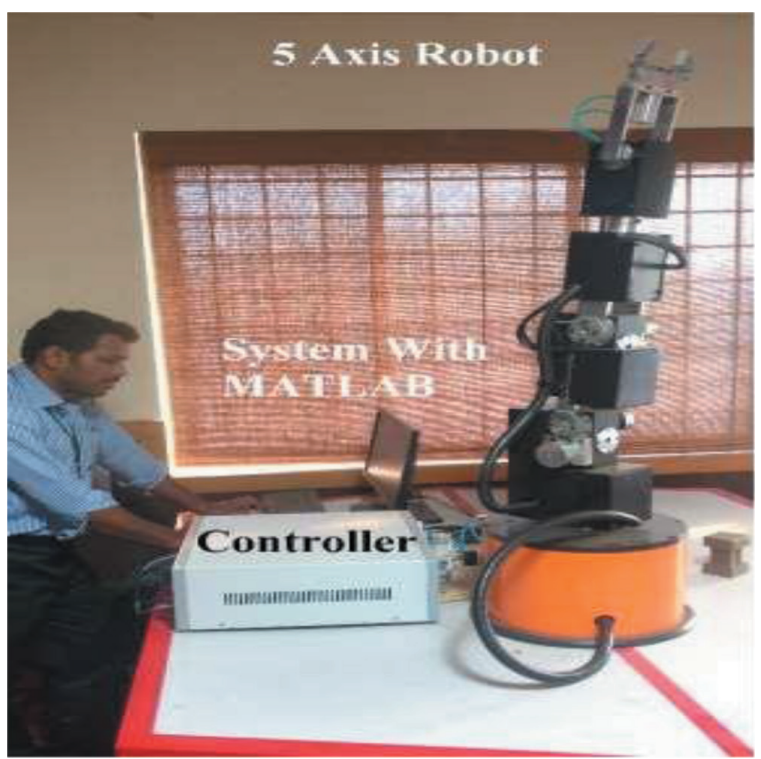

Figure 1. VI5SNS five-axis education robot.

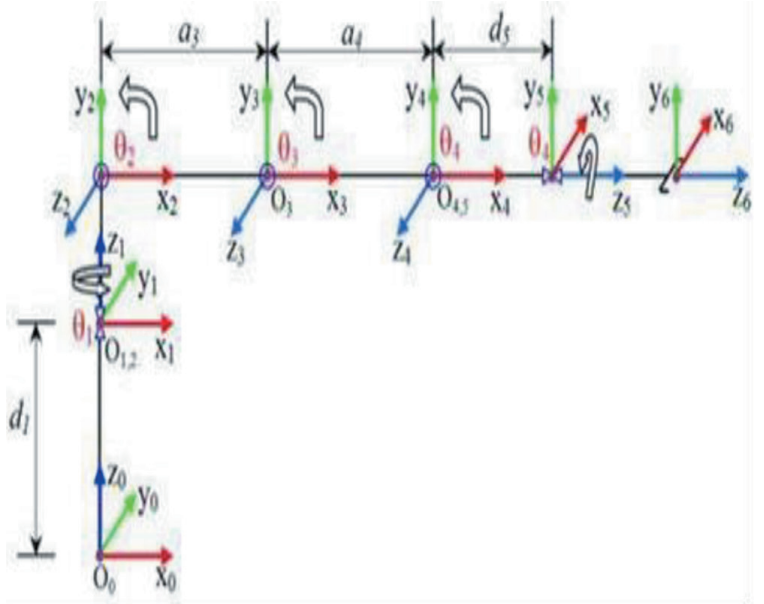

Figure 2. A rigid body in space to reference frame.

A graphical view of all of the joints changed into displayed in Figure 2. A rigid frame is completely defined in space by its function to a reference body (translation) and its orientation VI5SNS robot has rotational joints and the end effectors which are managed by way of dedicated servo motors. These automobiles are linked to a serial servo controller card to manipulate the VI5SNS from a laptop via the serial port $\mathrm{t}^{10}$.

\section{Robot Kinematics}

In order to calculate direct kinematics many methods can be adopted, the Denavit-Hartenberg analyses are one of the most used, on this method the direct kinematics is 
determinate from some parameters that must be defined, depending on each mechanism. However, it became chosen to apply the homogeneous transformation matrix. This transformation specifies the vicinity (role and orientation) of the hand in space with respect to the bottom of the robotic, but it does no longer inform us which configuration of the arm is required to acquire this place ${ }^{\underline{11}}$.

D-H parameters for VI5SNS are defined for the assigned frames in Table 1. For example Frame five is the grip frame with attached end effectors at joint 5 .

Table 1. DH parameter of VI5SNS robot

\begin{tabular}{|c|c|c|c|c|}
\hline I & $\boldsymbol{\alpha i}-\mathbf{1}$ & $\mathbf{a i}-\mathbf{1}$ & $\mathbf{d i}$ & $\boldsymbol{\theta i}$ \\
\hline 1 & 0 & 0 & $\mathrm{~d} 1$ & $\theta 1^{*}$ \\
\hline 2 & 90 & 0 & 0 & $\theta 2^{*}$ \\
\hline 3 & 0 & $\mathrm{a} 3$ & 0 & $\theta 3^{*}$ \\
\hline 4 & 0 & $\mathrm{a} 4$ & 0 & $\theta 4-90^{*}$ \\
\hline 5 & -90 & 0 & $\mathrm{~d} 5$ & $\theta 5^{*}$ \\
\hline 6 & 0 & 0 & 0 & Gripper \\
\hline
\end{tabular}

By substituting these parameters; the transformation matrices T1 to T6 can be obtained as shown below. For example, $\mathrm{T} 1$ shows the transformation between frames 0 and 1 (designating $\mathrm{Ci}$ as $\cos \theta \mathrm{i}$ and $\mathrm{Si}$ as $\sin \theta \mathrm{ietc}$ ).

$$
\begin{array}{r}
T_{3}^{2}=\left[\begin{array}{ccl}
C \theta i & -S \theta i & 0 \\
S \theta i & C \theta i & 0 \\
0 & 0 & 1 \\
& & \\
C \theta i & -S \theta i & 0 \\
T_{5}^{4}=\left[\begin{array}{ccl}
0 & 0 & 1
\end{array}\right] \\
S \theta i & C \theta i & 0
\end{array}\right.
\end{array}
$$$$
\begin{array}{rcl}
C \theta i & -S \theta i & 0 \\
T^{3}=\left[\begin{array}{ccl}
S \theta i & C \theta i & 0
\end{array}\right] \\
0 & 0 & 1 \\
& & \\
C \theta i & -S \theta i & 0 \\
T_{\text {Gripper }}=\left[\begin{array}{ccc}
S \theta i & C \theta i & 0
\end{array}\right] \\
0 & 0 & 1
\end{array}
$$

\section{Forward Kinematic}

Calculating the position and orientation of the give up effectors with given joint angles is known as forwarding kinematics analysis. Forward Kinematics equations are generated from the transformation matrixes shown underneath and the ahead kinematics answer of the arm is the product of these six matrices identified as 0T6 (with recognizing to base) proven in Equation (1) $)^{12}$

The first three columns inside the matrices constitute the orientation of the give up effectors, whereas the remaining column represents the position of the give up effectors. The orientation and role of the end effectors may be calculated in terms of joint angles ${ }^{13}$.

\section{Inverse Kinematic}

Inverse Kinematics evaluation determines the joint angles for preferred position and orientation in Cartesian area. Total Transformation matrix Equation can be used to calculate inverse kinematics equations. Its solution, but, is much more complicated than direct kinematics given that there is no particular analytical answer. Each manipulator needs a specific approach considering the machine structure and regulations $\boldsymbol{s}^{\underline{14}}$.

\section{Geometric Approach}

Using IK-Cartesian mode, the consumer specifies the favoured target role of the gripper in Cartesian area as ( $\mathrm{x}$, $\mathrm{y}, \mathrm{z}$ ) wherein $\mathrm{z}$ is the height, and the perspective of the gripper relative to ground, $\psi$ (This steady $\psi$ allows users to move items without changing the item's orientation (the keeping a cup of liquid scenario). In addition, via either preserving $\psi$ constant in position mode or preserving the wrist fixed relative to the rest of the arm, the inverse kinematic equations can be solved in closed form as we now show for the case of a hard and fast $\psi^{15}$.

The lengths $\mathrm{d}, \mathrm{a}$, a and $\mathrm{d}$ correspond to the bottom top, higher arm period, forearm duration and gripper length, respectively and are regular. The angles $\theta 1, \theta 2, \theta 3$, $\theta 4$ and $\theta$. Correspond to shoulder rotation, upper arm, and forearm, wrist, and End effectors, respectively. These angles are up to date as the desired position in space adjustments ${ }^{16}$.

We remedy for the joint angles of the arm, $\theta 1: 4$

Given preferred position ( $\mathrm{x}, \mathrm{y}$, and $\mathrm{z}$ ) and $\psi$ which might be inserted by way of the user. From Figure 3, we clearly see that

$\theta 1=A \tan 2(y, x)$ and the specified radial distance from the bottom $\mathrm{d}$ are related to $\mathrm{x}$ and $\mathrm{y}$ with the aid of

$$
\begin{aligned}
& =\sqrt{x_{d}{ }^{2}+{ }_{d}^{2}} \\
& =d \cos \theta 1 \\
& =d \sin \theta 1
\end{aligned}
$$

Moving now to the planar view in Figure. Three, we discover a relationship among joint angles $\theta 2, \theta 3$ and $\theta 4$ and $\psi$ as follows:

$$
\varphi=\theta 2+\theta 3+\theta 4
$$

Since $\psi$ is given, we will calculate the radial distance and peak of the wrist joint:

$$
\begin{aligned}
& r 4=r d-a 5 \cos \varphi \\
& z 4=z d-a 5 \sin \varphi
\end{aligned}
$$




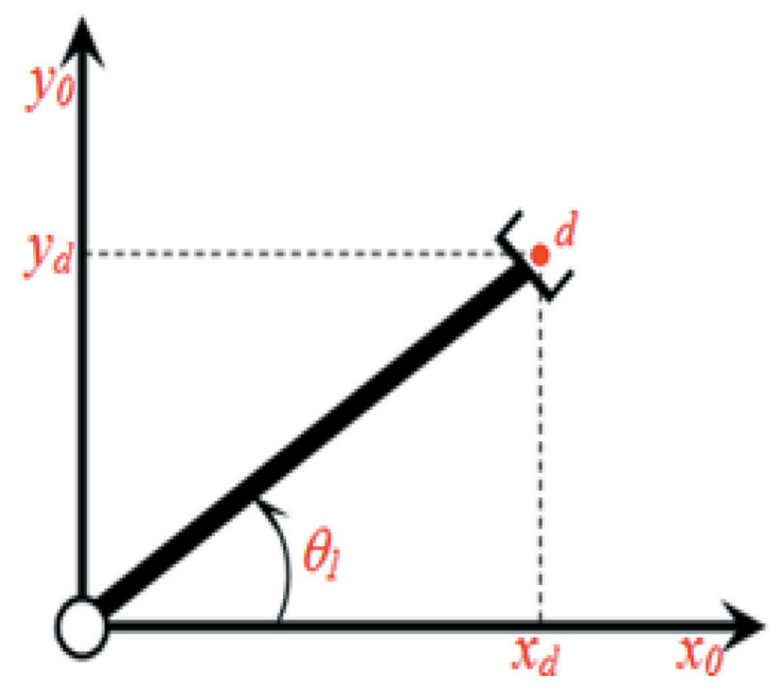

Figure 3. Planar view.

With those intermediate values from Equations (2), (3), (4), (5), (6) and (7) we can now find the remaining angle.

\section{Velocity Kinematics/Arm Jacobian}

The Jacobian is one of the maximum important quantities inside the evaluation and manipulates of robotic movement. It is used for clean trajectory making plans and execution inside the derivation of the dynamic equation. To look at target with specified velocity, every joint speed at the required joint positions desires to be discovered. This is achieved using Jacobian, which is used to narrate joint velocities to the linear and angular velocities of the end-effector ${ }^{17}$.

\section{Trajectory Planning}

Trajectory planning approximates the favored course by using a class of polynomial capabilities and generates a sequence of time-primarily based "control set factors" for the manipulate of manipulator from the preliminary configuration to its vacation spot. Suppose that we wish to generate a trajectory among two configurations, and that we desire to specify the start and end velocities for the trajectory. If we have four constraints to satisfy, including following equation we require a polynomial with four independent coefficients that may be chosen to meet those constraints ${ }^{18}$.

\section{Software}

The first step on this method is to design the arm in AutoCAD three-D application. The application selected for this was Autodesk Inventor. Autodesk Inventor lets in the arm to be designed and visualized at the same time. It also lets in the arm to be checked for feasible collisions and link interference. Because each link depends upon the previous link, the design of the arm needs to begin at the bottom and end on the cease effector or gripper ${ }^{19}$. Trunk or base is therefore the primary to be designed, observed through shoulder, and so forth. This means that the design method is fairly involved, as each hyperlink must be redesigned numerous instances ${ }^{20}$. After completed the photographs design by using the use of the AutoCAD 3D we connected it to MATLAB. We then use the CAD to Mat lab function, wherein this feature takes a CAD record in (.Stl or .Slp layout) and converts the identical into Mat lab supporting documents. We want to apply anther application to convert the AutoCAD dwg to slip record like PolyTrans 3-D from Okino Computer Graphics, by using the usage of this program we will modifying the robot link and coordinate and keep the document as slp ${ }^{21}$.

Software package deal was developed to compute the ahead kinematics, inverse kinematics, Jacobian, trajectory planning of VI5SNS robot. Graphical User Interface (GUI) Development Platform with Mat Lab programming language turned into used for implementation. An On-line motional simulator of the robotic arm become

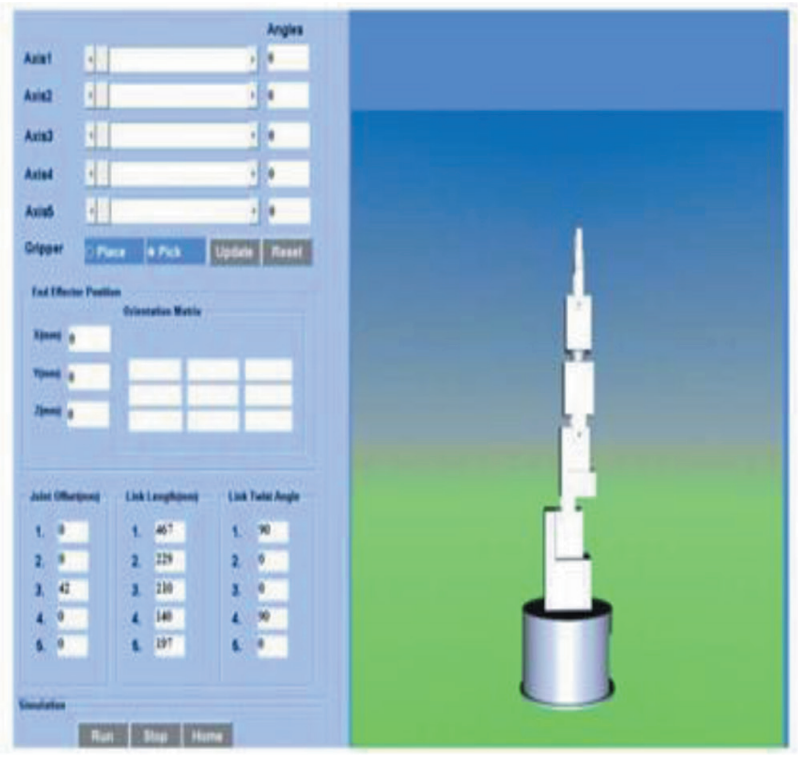

Figure 4. Graphical user interface of VI5SNS. 
additionally covered with GUI to reveal the generated movement in Figure 7 based on the theoretical analysis provided in this paper.

\section{Results and Discussions}

Mathematical modeling and kinematic analysis of VI5SNS, changed into developed and tested on this observe. Robot arm changed into mathematically modeled with Denavit-Hartenberg (D-H) approach. Forward, Inverse Kinematics, Jacobian and direction planning answers were generated and applied via the evolved software program.
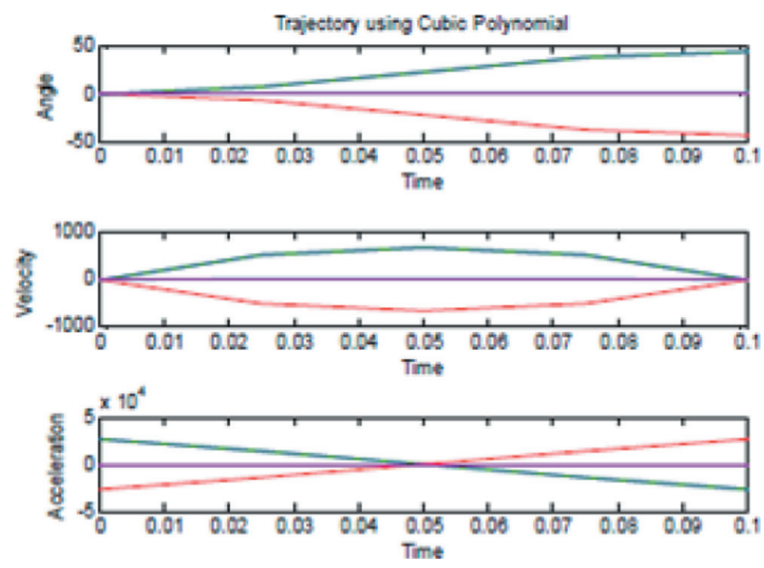

Figure 5. Trajectory planning of VI5SNS robot.

After trying out the ahead kinematic as example for the favoured function $\mathrm{x}, \mathrm{y}$ and $\mathrm{z}$ (199.Three, 199.Three, 154 . Nine) the input angles $\boldsymbol{\theta}(1,2$, three, 4 , five) identical (forty five, 45 ,-forty five, zero, zero) and Vis versa for inverse kinematic as shown in Figure 5. A entire Kinematics evaluation of the VI5SNS robot turned into investigated. Graphical User Interface (GUI) become developed to test and simulate the motional characteristics of the Robot. A physical interface between the VI5SNS robot and the GUI could be designed. The advanced machine might be diagnosed as an educational experimental device; it is able to be utilized in graduate and undergraduate robotic publications to recognise the relationships between theoretical and realistic components of robot manipulator motions in real time.

\section{Acknowledgments}

We like to thank management of SNS College of Technology, Coimbatore for giving us area and robot to analysis its features and working.

\section{References}

1. Piltan F, Emamzadeh S, Hivand Z, Shahriyari F, Mirzaei M. PUMA-560 robot manipulator position sliding mode control methods using Matlab/Simulink and their integration into graduate/undergraduate nonlinear control, robotics and MATLAB courses. International Journal of Robotic and Automation. 2012; 6(3):167-91.

2. Delavari H, Ghaderi R, Ranjbar AN, Hossein Nia SH, Momani S. Adaptive fractional PID controller for robot manipulator. The 4th IFAC Workshop Fractional Differentiation and its Applications; Badajoz, Spain; 2010. p. 1-7.

3. Yadegar S, Soh ABC. Design stable robust intelligent nonlinear controller for 6- DOF serial links robot manipulator. International Journal of Intelligent Systems and Applications (IJISA) MECS. 2014. p. 19-38. https://doi.org/10.5815/ijisa.2014.08.03

4. Aung CH, Lwin KT, Myint YM. Modeling motion control system for motorized robot arm using Matlab. Proceedings of World Academy of Science, Engineering And Technology. 2008; 32.

5. Arimoto S, Nguyen PTA, Han H-Y, Doulgeri Z. Dynamics and control of aset of dualfingerswith soft tips. Robotica. 2000; 18(1)Part 1:71 $\quad-80$. https://doi.org/10.1017/S0263574799002441

6. Nguyen PTA, Arimoto S, Han H-Y. Computer simulation of dynamics of dual fingers with soft-tips grasping an object. Ann Arbor, Michigan; 2000.

7. Arimoto S. Control theory of nonlinear mechanical systems: A passivity-based and circuit-theoretic approach. Oxford, UK: Oxford University Press; 1996.

8. Raibert $\mathrm{MH}$, Craig JJ. Hybrid position/force control of manipulators. ASME J Dyn Syst Meas Control. 1981; 103(2):126-33. https://doi.org/10.1115/1.3139652

9. Nilsson K, Johansson R. Integrated architecture for industrial robot programming and control. J Robot Auton Syst. 1999; 29(4):205-26. https://doi.org/10.1016/S09218890(99)00056-1

10. Bruyninckx H. Open robot control software: The OROCOS project. Proc Int Conf Robotics and Automation. 2001; 3:2523-8. https://doi.org/10.1109/ROBOT.2001.933002

11. Born H, Bunsendal J. Programmable multi-sensor interface for industrial applications. Proc Int Conf on Multisensor Fusion and Integration for Intell Syst; 2001. p. 189-94.

12. Yoshikawa T. Force control of robot manipulators. Proc IEEE Int Conf on Robotics and Automation; 2000. p. 220-6.

13. Kanehiro F, Hirukawa H, Kajita S. Open HRP: Open architecture humanoid robotics platform. Int J of Robotics Research. 2004; 23:155-65. https://doi. org/10.1177/0278364904041324 
14. Koenig N, Howard A. Design and use paradigms for Gazebo, an open-source multi-robot simulator. Proc of Int Conf on Intelligent Robots and Systems; 2004. p. 2149-54.

15. Quigley M, Gerkeyy B, Conleyy K, Fausty J, Footey T, Leibsz J, Bergery E, Wheelery R, Ng A. ROS: An opensource robot operating system. Proc of IEEE Int Conf of Robotics and Automation; 2009.

16. Freese M, Ozaki F, Matsuhira N. Collision detection, distance calculation and proximity sensor simulation using oriented bounding box trees. 4th International Conference on Advanced Mechatronics; 2004. p. 13-8. https://doi.org/10.1299/jsmeicam.2004.4.19_3

17. Wampler CW. Manipulator inverse kinematic solutions based on vector formulations and damped least squares methods. IEEE Trans Syst Man Cybern 1986; 16:93-101. https://doi.org/10.1109/TSMC.1986.289285
18. Gottschalk S, Lin MC, Manocha D. OBB-tree: A hierarchical structure for rapid interference detection. ACM SIGGRAPH; 1996. p. 171180. https://doi. org/10.1145/237170.237244 PMid:8829278

19. Pradeep S, Kousalya T, Suresh KMA, Edwin J. IoT and its connectivity challenges in smart home. IRJET. 2016 Dec; 3(12):1040-3.

20. Kousalya T, Pradeep S, Unnikrishna R, Pereira TJM. Study and implementation of fault diagnosis in induction motor using MCSA. International Research Journal of Advanced Engineering and Science. 2016; 1(4):199-201.

21. Yasodharan R, Sivabalakrishnan R, Devendran P. Trusted routing with an efficient certificate revocation for mobile ad hoc network. IJISET- International Journal of Innovative Science, Engineering and Technology. 2015; 2(3):755-62. 